\title{
SHAPE - A STOCHASTIC HYBRID APPROXIMATION PROCEDURE FOR TWO-STAGE STOCHASTIC PROGRAMS
}

\author{
RAYMOND K.-M. CHEUNG \\ Department of Industrial Engineering and Engineering Management, Hong Kong University of Science and Technology, \\ Clearwater Bay, Hong Kong
}

WARREN B. POWELL

Department of Civil Engineering and Operations Research, Princeton University, Princeton, New Jersey 08544

(Received February 1995; revision received December 1995, April 1997; accepted July 1998)

\begin{abstract}
We consider the problem of approximating the expected recourse function for two-stage stochastic programs. Our problem is motivated by applications that have special structure, such as an underlying network that allows reasonable approximations to the expected recourse function to be developed. In this paper, we show how these approximations can be improved by combining them with sample gradient information from the true recourse function. For the case of strictly convex nonlinear approximations, we prove convergence for this hybrid approximation. The method is attractive for practical reasons because it retains the structure of the approximation.
\end{abstract}

A common problem in operations research is the challenge of making a decision now in a way that minimizes the expectation of costs in the future that depend on random events. For example, we may have the situation of determining how much product to ship from plants to warehouses from which we then satisfy demands at different retailers. We must decide how much product to ship to each warehouse before we know the retail demand. Once retail demands are known, we are able to optimize shipping patterns between warehouses and retailers.

This problem, and many like it, can be posed as two-stage stochastic programs. The decision made now (in stage 1) determines what state we are in when we have to solve the problem in stage 2 . If we could exactly capture the structure of the expected cost function (or recourse function) for stage 2 , we would be able to make optimal decisions now. The difficulty is that in most cases, the structure of this expected cost function is too complex.

There is an extensive literature on two-stage stochastic programming problems, which is nicely summarized in several recent books (Infanger 1994 and Kall and Wallace 1994). General solution methods include scenario optimization (e.g., Rockafellar and Wets 1991), stochastic gradient techniques (e.g., Ermoliev 1983, Ruszczynski 1980), Benders decomposition and its variants (e.g., Van Slyke and Wets 1969, Birge 1985, and Higle and Sen 1991), sample path optimization (Robinson 1996), and other approximation techniques (e.g., Beale et al. 1980). These techniques are, for the most part, very general and are not designed specifically to take advantage of approximations that may produce good but not optimal solutions.
In this paper, we propose a new algorithm called SHAPE (successive hybrid approximation procedure) that combines an initial nonlinear approximation with iteratively sampled stochastic gradient information. The initial nonlinear approximation can exploit problem structure, while the stochastic gradient information, which is easy to obtain for most problems, tunes the approximation. We describe the algorithm, provide a small numerical illustration, and prove convergence.

Section 1 provides a formal problem statement and introduces the basic idea behind the algorithm. Section 2 presents a method we call the stochastic hybrid approximation procedure (SHAPE) for solving two-stage stochastic programs with recourse. Section 3 presents the proof of convergence of SHAPE. Finally, $\S 4$ summarizes the results of this paper and discusses areas of application and further research.

\section{THE BASIC IDEA}

Consider a two-stage stochastic programming problem which can be stated as

$\min _{x, S} c_{0}^{T} x+E_{\omega}[Q(S, \omega)]$

subject to:

$A_{0} x=b_{0}$,

$B_{0} x-S=0$,

$x \leqslant u_{0}$

where the recourse function $Q(S, \omega)$ is given by

$Q(S, \omega)=\min _{y(\omega)} c_{1}^{T} y(\omega)$ 
subject to:

$A_{1}(\omega) y(\omega)=b_{1}(\omega)+S$,

$y(\omega) \leqslant u_{1}(\omega)$.

In this model, Equations (1)-(4) define the first stage problem and Equations (5)-(7) define the second stage problem. The variables $x$ and $y$ are the decision variables of the stage 1 and stage 2 problems, respectively; whereas the parameters $c_{0}$ and $c_{1}$ represent the first and second stage vectors of cost coefficients, $A_{0}, B_{0}, A_{1}$ are constraint matrices, and $u_{0}$ and $u_{1}$ are the upper bounds of the decision variables. Note that the second stage problem is defined for an outcome $\omega \in \Omega$ from a probability space $(\Omega, \mathscr{F}, P)$. Finally, actions $x$ in the first stage are communicated to the second stage through the state variable $S=\left(S_{1}, S_{2}, \ldots, S_{m}\right)$, which is characteristically of much lower dimensionality than $x$. We write the minimization in (1) as a function of $x$ and $S$, although $S$ is defined as a function of $x$ as given in (3).

Our research is motivated by a problem arising in transportation involving the management of large fleets of vehicles, referred to as the dynamic vehicle allocation problem. Powell (1988) shows how this problem can be modeled as a multistage network with random arc capacities. The random arc capacities represent forecasted demands that are uncertain. In a typical application, a single stage of the problem might exhibit thousands of random variables, and the state vector $S$ might have dimensionality on the order of 100 .

For this application, $A_{0}, B_{0}$, and $A_{1}$ are node-arc incidence matrices. Given the large size of the problem, there is a strong desire to take advantage of the underlying network structure. Prior efforts at solving this problem (Frantzeskakis and Powell 1990, Powell 1988, and Cheung and Powell 1996) have focused on replacing the expected recourse function in Equation (1) with a piecewise linear, separable function $\hat{Q}(S)$, which can be written as

$\hat{Q}(S)=\sum_{i=1}^{m} \hat{Q}_{i}\left(S_{i}\right)$.

If the approximate functions $\hat{Q}_{i}\left(S_{i}\right)$ are piecewise linear, then we can replace the expected recourse function in (1) with $\hat{Q}(S)$ and solve the approximation as a pure network. In general, this approach cannot produce an optimal solution. Furthermore, replacing a nonseparable function with a separable one will introduce errors, raising the question of whether the approximation can be improved in some way.

An alternative approach is to simply replace the recourse function with a stochastic subgradient (note that the recourse function is convex and continuous, but can be piecewise linear), and apply the theory of stochastic subgradients (Ermoliev 1988 and Ruszczynski 1987). Thus, instead of replacing the recourse function with a nonlinear, separable approximation, we replace it with a linear (and therefore separable) approximation. The result would require iteratively solving problems with the general form

$\min _{x, S} c_{0}^{T} x+\left(\bar{g}^{k}\right)^{T} S$ where $\bar{g}^{k}$ is a smoothed estimate of the gradient of the expected recourse function at iteration $k$. The linear approximation helps to retain special structure in the first stage. For example, if constraints (2)-(3) are network flow constraints, then problem (9) is also a network problem. Furthermore, the method is provably convergent. The drawback is that the algorithm is very slow and does little to take advantage of the structure of the recourse function.

In this paper, we would like to combine our ability to build accurate approximations like $\hat{Q}(S)$ in Equation (8) with the convergence properties of stochastic subgradient methods. We propose to combine Equations (1), (8), and (9) to form an approximation that at iteration $k$ would be

$\min _{x, S} c_{0}^{T} x+\hat{Q}^{0}(S)+\left(\bar{g}^{k}\right)^{T} S$.

In this model, we combine a nonlinear, differentiable approximation $\hat{Q}^{0}(S)$ with a linear correction term $\left(\bar{g}^{k}\right)^{T} S$. With this formulation, we take advantage of the fact that if the problem has special structure (as often occurs with networks), we can construct an initial approximation $\hat{Q}^{0}(S)$ that is separable in a vector $S$ (which is related linearly to $x)$. We then use a linear correction term $\left(\bar{g}^{k}\right)^{T} S$ to correct for errors in $\hat{Q}^{0}(S)$. However, we do not calculate $\bar{g}^{k}$ in the usual manner used for calculating stochastic subgradients. Instead, we solve problems of the form

$\min _{x, S} c_{0}^{T} x+\hat{Q}^{k}(S)+a_{k}\left(g^{k}-\hat{q}^{k}\right)^{T} S$,

where $\hat{q}^{k}$ is a gradient of $\hat{Q}^{k}(\cdot)$ at a point $S$ and $\hat{Q}^{k}(S)$ is updated using the iteration

$\hat{Q}^{k+1}(S)=\hat{Q}^{k}(S)+a_{k}\left(g^{k}-\hat{q}^{k}\right)^{T} S$,

where $g^{k}$ is a stochastic subgradient of $Q(S, \omega)$. As shown later, $\bar{g}^{k}$ in (10) is a linear combination of $g^{1}, g^{2}, \ldots, g^{k}$. We show below that this updating mechanism is equivalent to solving problems with the same structure as Equation (10), where $\hat{Q}^{0}(S)$ is an initial approximation of the problem such as that given in Equation (8).

\section{A STOCHASTIC HYBRID APPROXIMATION PROCEDURE}

Consider a general two-stage stochastic program

$\min _{x \in \mathscr{X}} c_{0}^{T} x+E_{\omega} Q(x, \omega)$,

where $\mathscr{X} \subset \mathfrak{R}^{n}$ is a convex compact set and the recourse function $Q(x, \omega)$ is defined by

$Q(x, \omega)=\min _{y(\omega) \in \mathscr{Y}(x, \omega)} c_{1}^{T} y(\omega)$,

where $\mathscr{Y}(x, \omega)=\{y \mid W(\omega) y=h(\omega)-T(\omega) x\} \subset \mathfrak{R}^{m}$ is a feasible set for outcome $\omega$ in the second stage. We assume that problem (13) has relatively complete recourse. That is, $Q(x, \omega)$ is finite almost surely for any given feasible $x$ in stage 1 . In most dynamic network models, this assumption is valid. 
The stochastic hybrid approximation method is best illustrated by starting from the standard problem

$$
\min _{x \in \mathscr{X}} \bar{Q}(x)=E_{\omega} Q(x, \omega),
$$

where $\mathscr{X}$ is a convex, compact feasible set (we drop the term $c^{T} x$ for succinctness). To illustrate the idea, assume that the objective function $\bar{Q}(x)$ is bounded and continuous. Suppose that we are given an initial approximation of the expected recourse function, denoted by $\hat{Q}^{0}(x)$, which is bounded and differentiable. We begin by solving problem (14) with the function $\hat{Q}^{0}(x)$ and get the initial solution $x^{0}$. Next, we draw a realization of the random quantity, solve the resulting deterministic problem, and obtain a stochastic subgradient of $\bar{Q}(x)$. We then compare the slope of the stochastic subgradient with the slope of $\hat{Q}^{0}(x)$ at $x=x^{0}$. The difference of the slopes is used as a linear term to update $\hat{Q}^{0}(x)$. Finally, we use the updated approximation to obtain a new solution and then repeat the whole process again.

This method combines the nonlinear approximation approach with stochastic subgradients. Hence, we refer to it as the stochastic hybrid approximation procedure (SHAPE). To present this algorithm mathematically, we let, at iteration $k$ :

$$
\begin{aligned}
a_{k}= & \mathrm{a}(\text { possibly random }) \text { positive step-size; } \\
g^{k}= & \text { a stochastic subgradient of } \bar{Q}(x) \text { at } x^{k}, \\
& \text { that is, } g^{k} \in \partial Q\left(x^{k}, \omega^{k+1}\right) ;
\end{aligned}
$$

$\hat{Q}^{k}(x)=$ a convex differentiable approximation of $\bar{Q}(x)$;

$\hat{q}^{k}(x)=$ the gradient of $\hat{Q}^{k}(\cdot)$ at $x$.

We assume that a convex function $\hat{Q}^{0}(x)$ is available based on the structure of the problem. Then, we generate a sequence of solutions $x^{k}$ according to the following steps.

Algorithm:. SHAPE

Step 1. Set $k=0$ and obtain a strongly convex function $\hat{Q}^{0}(x)$;

Step 2. Obtain $x^{k}$ by

$$
x^{k}=\arg \min _{x \in \mathscr{X}} \hat{Q}^{k}(x)
$$

Step 3. Obtain $g^{k}$ and update $\hat{Q}^{k}(x)$ by

$$
\hat{Q}^{k+1}(x)=\hat{Q}^{k}(x)+a_{k}\left(g^{k}-\hat{q}^{k}\left(x^{k}\right)\right)^{T} x ;
$$

Step 4. Check for convergence (for example, an improvement in $\hat{Q}^{k}(x)$ within the last $K$ iterations). Failing this check, set $k=k+1$ and go to Step 2 .

As is common with algorithms of this type, the convergence check is heuristic in nature. While we seek theoretical guarantees that the algorithm will eventually converge, in practice, we resort to approximate rules.
Below we provide a simple illustration of how and why the method works, followed in $\S 3$ by a proof of convergence.

The algorithm SHAPE is similar in spirit to the auxiliary function method of Culioli and Cohen (1990). Their method solves sequences of problems with the form

$x^{k+1}=\arg \min _{x \in \mathscr{X}} K(x)+\left(a_{k} g^{k}-\nabla K\left(x^{k}\right)\right)^{T} x$,

where $K(x)$ is a strongly convex function, and $\nabla K\left(x^{k}\right)$ is the gradient of $K(x)$ at $x^{k}$. In this case, $K(x)$ is a general nonlinear function with no particular relationship to the structure of the problem.

It is useful to contrast the auxiliary function method with SHAPE. If we compare Equation (17) to Equation (16), we can observe that the main difference between the auxiliary function method and SHAPE is that the function $K(x)$ in (17) is not updated in the optimization process, while the function $\hat{Q}^{k}(x)$ in SHAPE is updated iteratively. The advantage of updating $\hat{Q}^{k}(x)$ is that the information from the stochastic subgradients used in the previous iterations can be retained. In other words, the objective function in iteration $k$ of the auxiliary function method involves only the stochastic subgradient obtained in iteration $k$; but the one used in SHAPE involves a weighted average of the stochastic subgradients in the past $k-1$ st iterations. Therefore, the difference between the auxiliary function method and SHAPE is similar to that between the steepest descent method and the conjugate gradient method in deterministic optimization.

\section{An Example}

To illustrate a step of the hybrid method, we consider the following deterministic minimization problem:

$\min _{x \in \Re} \bar{Q}(x)=\frac{1}{2} x^{2}-2 x$.

Obviously, the optimal value of problem (2) is attained when $x=2$. Suppose we are given an initial convex approximation $\hat{Q}^{0}(x)$ :

$\hat{Q}^{0}(x)=\frac{2}{3} x^{2}-2 x$,

which is plotted in Figure 1a. We begin by obtaining the initial solution $x^{0}$ :

$x^{0}=\arg \min _{x \in \Re} \frac{2}{3} x^{2}-2 x=\frac{3}{2}$.

Assume that we obtain a gradient $g^{0}=-\frac{1}{2}$ at $x^{0}=\frac{3}{2}$, as shown in Figure 1a. Then, with step-size $a_{0}=\frac{3}{4}$, the updated approximation is

$$
\begin{aligned}
\hat{Q}^{1}(x) & =\hat{Q}^{0}(x)+a_{k}\left(g^{0}-\hat{q}^{0}\left(x^{0}\right)\right) x \\
& =\frac{2}{3} x^{2}-\frac{19}{8} x .
\end{aligned}
$$

When we minimize $\hat{Q}^{1}(x)$, we obtain $x^{1}=\frac{57}{32}$. The updated approximation $\hat{Q}^{1}(x)$ and $\bar{Q}(x)$ are plotted in Figure $1 \mathrm{~b}$. 
Figure 1. A step in the hybrid method.

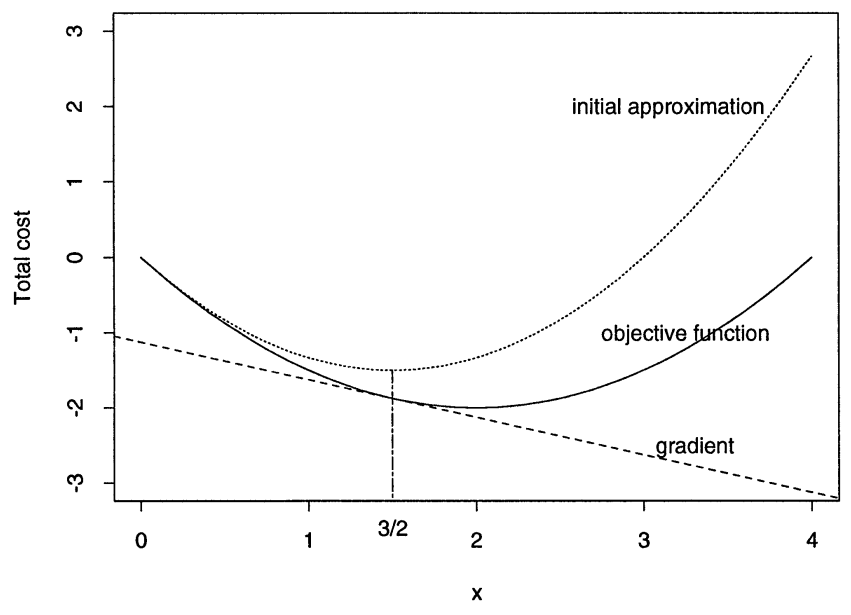

(a) Initial approximation

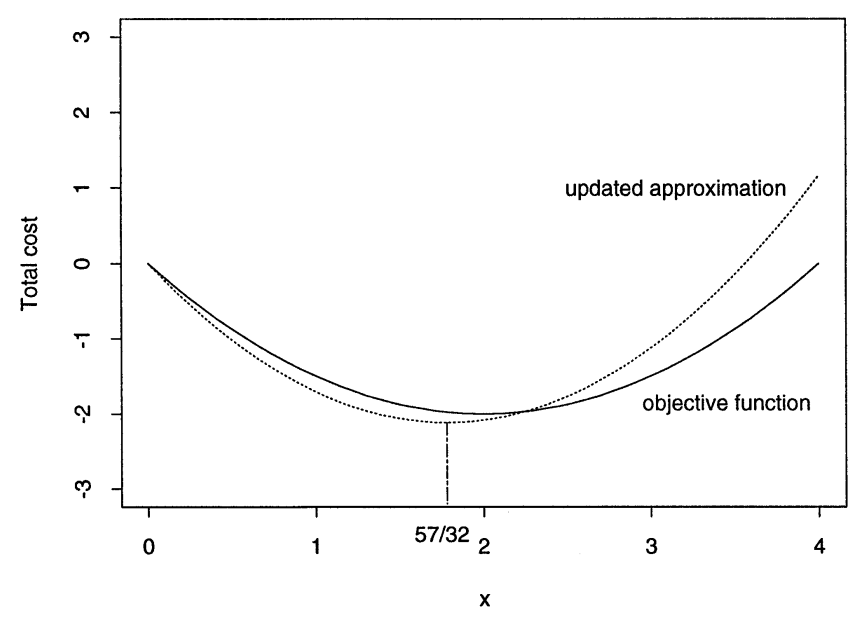

(b) Updated approximation

Figure 1a shows that the gradient $g^{0}$ serves as a linear correction term that tilts the initial convex approximation $\hat{Q}^{0}(x)$. In particular, Figure $1 \mathrm{~b}$ shows that the shape of the updated approximation $\hat{Q}^{1}(x)$ comes closer to that of $\bar{Q}(x)$. This example illustrates the solution of a deterministic problem. In a stochastic context, $g$ would be based on a realization of the recourse function.

\section{CONVERGENCE OF SHAPE}

In this section, we first state the convergence theorem of SHAPE. Second, we state the Martingale convergence theorem (see Doob 1953, Neveu 1975, and Taylor 1990 for details) that has been used to prove the convergence of some stochastic subgradient methods (see, for example, Gladyshev 1965). Third, we list some properties of the approximations $\hat{Q}(\cdot)$ and establish a bound on the changes of two consecutive $x^{k}$. Finally, we provide the proof of our theorem. We let $\mathscr{H}_{k}=\omega_{0}, \omega_{1}, \ldots, \omega_{k}$ be the history up to (and including) iteration $k$.

\section{Convergence Theorem of SHAPE}

We assume the following:

(A.1) $\mathscr{X}$ is convex and compact.

(A.2) $E_{\omega} Q(x, \omega)$ is convex, finite and continuous on $\mathscr{X}$.

(A.3) $g^{k}$ is bounded such that $\left\|g^{k}\right\| \leqslant c_{1}$ for each $\omega \in \Omega$.

(A.4) $\hat{Q}^{k}(x)$ is strongly convex, meaning that

$\hat{Q}^{k}(y)-\hat{Q}^{k}(x) \geqslant \hat{q}^{k}(x)^{T}(y-x)+b\|x-y\|^{2}$,

where $b$ is a positive number which is a constant throughout the optimization process. The term $b\|x-y\|^{2}$ is used to ensure that the slope $\hat{q}^{k}(x)$ is a monotone function of $x$. When we interchange $y$ and $x$ in (18) and add the resulting inequality to (18), we can write

$$
\left(\hat{q}^{k}(y)-\hat{q}^{k}(x)\right)^{T}(y-x) \geqslant 2 b\|x-y\|^{2} .
$$

(A.5) The stepsizes $a_{k}$ are $\mathscr{H}_{k}$ measurable and satisfy

$$
0<a_{k}<1, \quad \sum_{k=0}^{\infty} E\left\{a_{k}^{2}\right\}<\infty
$$

and

$$
\sum_{k=0}^{\infty} a_{k}=\infty \quad \text { a.s. }
$$

(A.6) $\hat{Q}^{0}(k)$ is bounded and continuous, and $\hat{q}^{0}(x)$ (the derivative of $\left.\hat{Q}^{0}(x)\right)$ is bounded for $x \in \mathscr{X}$.

Note that we require that the expected sum of squares be bounded, whereas we must impose the almost sure condition that the sum of stepsizes be infinite. We now state our primary theory.

Theorem 1. If (A.1)-(A.6) are satisfied, then the sequence $x^{k}$ produced by algorithm SHAPE converges almost surely to an optimal solution $x^{*} \in \mathscr{X}^{*}$ of problem (14).

To prove the theorem, we need to use the Martingale convergence theorem and two lemmas.

Martingale Convergence Theorem. A sequence of random variable $\left\{W^{k}\right\}$, which are $\mathscr{H}_{k}$-measurable, is said to be a supermartingale if the sequence of conditional expectations $E\left\{W^{k+1} \mid \mathscr{H}_{k}\right\}$ exists and satisfies

$E\left\{W^{k+1} \mid \mathscr{H}_{k}\right\} \leqslant W^{k}$.

TheOREM 2. (From Neveu 1975, p. 26) Let $W^{k}$ be a positive supermartingale. Then, $W^{k}$ converges to a finite random variable a.s. 
From the definition, $W^{k}$ is essentially the stochastic ana$\log$ of a decreasing sequence.

\section{Property of Approximations}

In addition to Equations (18)-(19) of Assumption (A.4), the optimal solution for problem (15) at iteration $k$ can be characterized by the variational inequality

$\hat{q}^{k}\left(x^{k}\right)^{T}\left(x-x^{k}\right) \geqslant 0 \quad \forall x \in \mathscr{X}$.

Furthermore, at iteration $k+1$.

$\left(\hat{q}^{k}\left(x^{k+1}\right)+a_{k}\left(g^{k}-\hat{q}^{k}\left(x^{k}\right)\right)\right)^{T}\left(x-x^{k+1}\right) \geqslant 0 \quad \forall x \in \mathscr{X}$.

The first of the two lemmas below shows that the difference between the solutions of two consecutive iterations is bounded by the stepsize and the magnitude of the stochastic gradient. The second lemma shows that the approximation $Q^{k}(x)$ is finite.

LEMMA 1. The solutions $x^{k}$ produced by algorithm SHAPE satisfy

$$
\left\|x^{k}-x^{k+1}\right\| \leqslant \frac{a_{k}}{2 b}\left\|g^{k}\right\|
$$

where b satisfies Equation (18).

Proof. Substituting $x$ by $x^{k}$ in (23), we have

$$
\begin{gathered}
a_{k}\left(g^{k}-\hat{q}^{k}\left(x^{k}\right)\right)^{T}\left(x^{k}-x^{k+1}\right) \\
\geqslant \hat{q}^{k}\left(x^{k+1}\right)^{T}\left(x^{k+1}-x^{k}\right) .
\end{gathered}
$$

Rearranging the terms, we obtain

$$
\begin{aligned}
& a_{k} g^{k^{T}}\left(x^{k}-x^{k+1}\right) \\
& \geqslant \hat{q}^{k}\left(x^{k+1}\right)^{T}\left(x^{k+1}-x^{k}\right)-a_{k} \hat{q}^{k}\left(x^{k+1}-x^{k}\right) \\
&=\left(\hat{q}^{k}\left(x^{k+1}\right)-\hat{q}^{k}\left(x^{k}\right)\right)^{T}\left(x^{k+1}-x^{k}\right) \\
&+\left(1-a_{k}\right) \hat{q}^{k}\left(x^{k}\right)^{T}\left(x^{k+1}-x^{k}\right) .
\end{aligned}
$$

Using Equations (19), (22), and $0<a_{k}<1$, we get

$$
\begin{aligned}
& a_{k} g^{k^{T}}\left(x^{k}-x^{k+1}\right) \\
& \quad \geqslant 2 b\left\|x^{k}-x^{k+1}\right\|^{2}+\left(1-a_{k}\right) \hat{q}^{k}\left(x^{k}\right)^{T}\left(x^{k+1}-x^{k}\right) \\
& \quad \geqslant 2 b\left\|x^{k}-x^{k+1}\right\|^{2} .
\end{aligned}
$$

Applying Schwarz's inequality, we have that

$$
\begin{aligned}
a_{k}\left\|g^{k}\right\| \cdot\left\|x^{k}-x^{k+1}\right\| & \geqslant a_{k} g^{k^{T}}\left(x^{k}-x^{k+1}\right) \\
& \geqslant 2 b\left\|x^{k}-x^{k+1}\right\|^{2} .
\end{aligned}
$$

Dividing both sides by $\left\|x^{k}-x^{k+1}\right\|$, it follows that $\left\|x^{k}-x^{k+1}\right\| \leqslant \frac{a_{k}}{2 b}\left\|g^{k}\right\|$.
Lemma 2. The approximation function $\hat{Q}^{k}(x)$ in iteration $k$ can be written as

$\hat{Q}^{k}(x)=\hat{Q}^{0}(x)+r^{k^{T}} x$

where $r^{k}$ is a finite vector.

Proof. Because only linear terms are added to the convex approximation in our algorithm, the approximation in iteration $k$ is the original function $\hat{Q}^{0}(x)$ plus a linear term

$\hat{Q}^{k}(x)=\hat{Q}^{0}(x)+r^{k^{T}} x$

where $r^{k}$ is the cumulative change of $\hat{Q}^{0}$ up to iteration $k$. It remains to show that $r^{k}$ is a finite vector for any $k$.

When taking the first derivative of $\hat{Q}^{k}(x)$ in Equation (25), we have

$\hat{q}^{k}(x)=\hat{q}^{0}(x)+r^{k}$.

With that, we can write $\hat{Q}^{k+1}(x)$ in terms of $\hat{Q}^{0}(x)$ :

$$
\begin{aligned}
\hat{Q}^{k+1}(x) & =\hat{Q}^{k}(x)+a_{k}\left(g^{k}-\hat{q}^{k}(x)\right)^{T} x, \\
& =\hat{Q}^{0}(x)+r^{k^{T}} x+a_{k}\left(g^{k}-\hat{q}^{k}(x)\right)^{T} x, \\
& =\hat{Q}^{0}(x)+r^{k^{T}} x+a_{k}\left(g^{k}-\hat{q}^{0}\left(x_{k}\right)-r^{k}\right)^{T} x .
\end{aligned}
$$

Therefore, $r^{k+1}$ and $r^{k}$ are related as follows:

$r^{k+1}=a_{k}\left(g^{k}-\hat{q}^{0}\left(x_{k}\right)\right)+\left(1-a_{k}\right) r^{k}$.

That is, the new cumulative change is a convex combination of the difference, $g^{k}-\hat{q}^{0}\left(x_{k}\right)$, and the current cumulative change. Since both $g^{k}$ and $\hat{q}^{0}\left(x_{k}\right)$ are finite, there exists a finite, positive vector such that

$\hat{d} \geqslant \max _{k}\left|g^{k}-\hat{q}^{0}\left(x^{k}\right)\right|$

where the inequality is applied componentwise. Next, we use induction to show that $r^{k} \leqslant \hat{d}$ for all $k$. For $k=1$, we have $r^{1}=a_{0}\left(g^{0}-\hat{q}_{0}^{0}\right) \leqslant a_{0} \hat{d}$. Since $a_{0}<1$ and is positive, we have $r^{1} \leqslant \hat{d}$. Assuming that $r^{k} \leqslant \hat{d}$, we want to show $r^{k+1} \leqslant \hat{d}$. By using this assumption and the definition of $\hat{d}$, Equation (30) implies that

$$
\begin{aligned}
r^{k+1} & \leqslant a_{k}\left|g^{k}-\hat{q}^{0}\left(x_{k}\right)\right|+\left(1-a_{k}\right) r^{k} \\
& \leqslant a_{k} \hat{d}+\left(1-a_{k}\right) \hat{d}=\hat{d}
\end{aligned}
$$

We now return to our main result.

Proof of Theorem 1. or simplicity, we write $\hat{q}^{k}=\hat{q}^{k}\left(x^{k}\right)$.

Step 1. Establish a supermartingale for Theorem 2.

Let $T^{k}=\hat{Q}^{k}\left(x^{*}\right)-\hat{Q}^{k}\left(x^{k}\right)$ and consider the difference of $T^{k+1}$ and $T^{k}$ :

$$
\begin{aligned}
T^{k+1}-T^{k}= & \hat{Q}^{k+1}\left(x^{*}\right)-\hat{Q}^{k+1}\left(x^{k+1}\right)-\hat{Q}^{k}\left(x^{*}\right)+\hat{Q}^{k}\left(x^{k}\right) \\
= & \hat{Q}^{k}\left(x^{*}\right)+a_{k}\left(g^{k}-\hat{q}^{k}\right)^{T} x^{*}-\hat{Q}^{k}\left(x^{k+1}\right) \\
& -a_{k}\left(g^{k}-\hat{q}^{k}\right)^{T} x^{k+1}-\hat{Q}^{k}\left(x^{*}\right)+\hat{Q}^{k}\left(x^{k}\right) .
\end{aligned}
$$


If we write $x^{*}-x^{k+1}$ as $x^{*}-x^{k}+x^{k}-x^{k+1}$, we get

$$
T^{k+1}-T^{k}=\underbrace{\hat{Q}^{k}\left(x^{k}\right)-\hat{Q}^{k}\left(x^{k+1}\right)-a_{k} \hat{q}^{k^{T}}\left(x^{k}-x^{k+1}\right)}
$$

$$
\begin{aligned}
& -\underbrace{a_{k} \hat{q}^{k^{T}}\left(x^{*}-x^{k}\right)}_{(\mathrm{II})}+\underbrace{a_{k} g^{k^{T}}\left(x^{*}-x^{k}\right)}_{(\mathrm{III})} \\
& +\underbrace{a_{k} g^{k^{T}}\left(x^{k}-x^{k+1}\right)}_{(\mathrm{IV})} .
\end{aligned}
$$

Consider each part individually. First, by convexity of $\hat{Q}^{k}(x)$, it follows that

$$
\begin{aligned}
\hat{Q}^{k}\left(x^{k}\right)-\hat{Q}^{k}\left(x^{k+1}\right) \leqslant & \hat{q}^{k^{T}}\left(x^{k}-x^{k+1}\right) \\
= & \left(1-a_{k}\right) \hat{q}^{k^{T}}\left(x^{k}-x^{k+1}\right) \\
& +a_{k} \hat{q}^{k^{T}}\left(x^{k}-x^{k+1}\right) .
\end{aligned}
$$

From Equation (22) and $0<a_{k}<1$, we know that $(\mathrm{I}) \leqslant 0$. Again from Equation (22) and $0<a_{k}<1$, we show that (II) $\geqslant 0$. For (III), by the definition that $g^{k} \in \partial Q\left(x^{k}, \omega^{k+1}\right)$,

$g^{k^{T}}\left(x^{*}-x^{k}\right) \leqslant Q\left(x^{*}, \omega^{k+1}\right)-Q\left(x^{k}, \omega^{k+1}\right)$,

where $Q\left(x, \omega^{k+1}\right)$ is the recourse function given outcome $\omega^{k+1}$. For (IV), Lemma 1 implies that

$$
\begin{aligned}
a_{k} g^{k^{T}}\left(x^{k}-x^{k+1}\right) & \leqslant a_{k}\left\|g^{k}\right\| \cdot\left\|x^{k}-x^{k+1}\right\| \\
& \leqslant \frac{a_{k}^{2}\left\|g^{k}\right\|^{2}}{2 b} \leqslant \frac{a_{k}^{2} c_{1}^{2}}{2 b} .
\end{aligned}
$$

Therefore, the difference $T^{k+1}-T^{k}$ becomes

$T^{k+1}-T^{k} \leqslant-a_{k}\left(Q\left(x^{k}, \omega^{k+1}\right)-Q\left(x^{*}, \omega^{k+1}\right)\right)+\frac{a_{k}^{2} c_{1}^{2}}{2 b}$.

Taking conditional expectations with respect to $\mathscr{H}_{k}$ on both sides, it follows that

$E\left\{T^{k+1} \mid \mathscr{H}_{k}\right\} \leqslant T^{k}-a_{k}\left(\bar{Q}\left(x^{k}\right)-\bar{Q}\left(x^{*}\right)\right)+\frac{a_{k}^{2} c_{1}^{2}}{2 b}$,

where $T^{k}, a_{k}$, and $x_{k}$ on the right-hand side are deterministic given the conditioning on $\mathscr{H}_{k}$. We have replaced $Q\left(x, \omega^{k+1}\right)$ (for $x=x^{k}$ and $x=x^{*}$ ) with its expectation $\bar{Q}(x)$ because conditioning on $\mathscr{H}_{k}$ tells us nothing about $\omega^{k+1}$. Since $\bar{Q}\left(x^{k}\right)-\bar{Q}\left(x^{*}\right) \geqslant 0$, the sequence

$W^{k}=T^{k}+\frac{c_{1}^{2}}{2 b} \sum_{i=k}^{\infty} a_{i}^{2}$

is a positive supermartingale. Theorem 2 implies the almost sure convergence of $W^{k}$. Thus,

$T^{k} \rightarrow T^{*} \quad$ a.s.

Step 2. Show that there exists a subsequence $k_{j}$ of $k$ such that $x^{k_{j}} \rightarrow x^{*} \in \mathscr{X}^{*}$ a.s.
Summing Equation (32) over $k$ up to $K$ and cancelling the alternating terms of $T^{k}$ gives

$$
\begin{aligned}
T^{K+1}-T^{0} \leqslant & -\sum_{k=0}^{K} a_{k}\left(Q\left(x^{k}, \omega^{k+1}\right)-Q\left(x^{*}, \omega^{k+1}\right)\right) \\
& +\sum_{k=0}^{K} \frac{a_{k}^{2} c_{1}^{2}}{2 b} .
\end{aligned}
$$

Take expectations of both sides. For the first term on the right-hand side, we first take the conditional expectation with respect to $\mathscr{H}_{k}$ and then over all $\mathscr{H}_{k}$ :

$$
\begin{aligned}
E\left\{T^{K+1}-T^{0}\right\} & \\
\leqslant & -\sum_{k=0}^{K} E\left\{E\left\{a_{k}\left(Q\left(x^{k}, \omega^{k+1}\right)-Q\left(x^{*}, \omega^{k+1}\right)\right) \mid \mathscr{H}_{k}\right\}\right\} \\
& +E\left\{\sum_{k=0}^{K} \frac{a_{k}^{2} c_{1}^{2}}{2 b}\right\} \\
\leqslant & -\sum_{k=0}^{K} E\left\{a_{k}\left(\bar{Q}\left(x^{k}\right)-\bar{Q}\left(x^{*}\right)\right)\right\}+\frac{c_{1}^{2}}{2 b} \sum_{k=0}^{K} E\left\{a_{k}^{2}\right\} .
\end{aligned}
$$

Taking the limit as $K \rightarrow \infty$ and using the finiteness of $T^{k}$ and $\sum_{i=0}^{\infty} E\left\{a_{i}^{2}\right\}$, we have

$$
\sum_{k=0}^{\infty} E\left\{a_{k}\left(\bar{Q}\left(x^{k}\right)-\bar{Q}\left(x^{*}\right)\right)\right\}<\infty .
$$

Because $\bar{Q}\left(x^{k}\right)-\bar{Q}\left(x^{*}\right) \geqslant 0$ and $\sum_{k=0}^{\infty} a_{k}=\infty$ (a.s.), there exists a subsequence $k_{j}$ of $k$ such that

$\bar{Q}\left(x^{k_{j}}\right) \rightarrow \bar{Q}\left(x^{*}\right)$ a.s.

By continuity of $\bar{Q}$, this sequence converges. That is,

$x^{k_{j}} \rightarrow x^{*} \in \mathscr{X}^{*} \quad$ a.s.

Step 3. Show that $x^{k} \rightarrow x^{*} \in \mathscr{X}^{*}$ a.s.

Consider the convergent subsequence $x^{k_{j}}$ in Step 2. By using the expression of $\hat{Q}^{k}$ in Lemma 2, we can write $T^{k_{j}}$ as

$$
\begin{aligned}
T^{k_{j}} & =\hat{Q}^{k_{j}}\left(x^{k_{j}}\right)-\hat{Q}^{k_{j}}\left(x^{*}\right) \\
& =\hat{Q}^{0}\left(x^{k_{j}}\right)-\hat{Q}^{0}\left(x^{*}\right)+r^{k_{j}^{T}}\left(x^{k_{j}}-x^{*}\right) \\
& \leqslant \hat{Q}^{0}\left(x^{k_{j}}\right)-\hat{Q}^{0}\left(x^{*}\right)+r^{k_{j}^{T}}\left|x^{k_{j}}-x^{*}\right| \\
& \leqslant \hat{Q}^{0}\left(x^{k_{j}}\right)-\hat{Q}^{0}\left(x^{*}\right)+\hat{d}^{T}\left|x^{k_{j}}-x^{*}\right|,
\end{aligned}
$$

where $\hat{d}$ is the positive, finite vector defined in (31). When $x^{k_{j}} \rightarrow x^{*}$, both the terms $\hat{d}^{T}\left|x^{k_{j}}-x^{*}\right|$ and $\hat{Q}^{0}\left(x^{k_{j}}\right)-\hat{Q}^{0}\left(x^{*}\right)$ (by continuity of $\hat{Q}^{0}$ ) go to 0 . Because $T^{k_{j}}$ is positive, we obtain that $T^{k_{j}} \rightarrow 0$ a.s. Combining this result and the result in Step 1 that $T^{k}$ converges to a unique nonnegative $T^{*}$ a.s., we have $T^{k} \rightarrow T^{*}=0$ a.s. Finally, we know from the strong convexity of $\hat{Q}^{k}(\cdot)$ that

$T^{k}=\hat{Q}^{k}\left(x^{k}\right)-\hat{Q}^{k}\left(x^{*}\right) \geqslant b\left\|x^{k}-x^{*}\right\|^{2} \geqslant 0$.

Therefore, $x^{k} \rightarrow x^{*}$ a.s. 


\section{SUMMARY}

This paper introduces a new method for solving two-stage stochastic programs by combining an initial nonlinear approximation with iteratively calculated stochastic gradients. The motivation of the method is that many problems exhibit special structure that suggest good approximations that will yield high quality, but not optimal, solutions. The SHAPE algorithm provides a way for combining these initial approximations with precise gradient information, producing a convergent algorithm without any loss of problem structure.

One particular feature of SHAPE is that we do not at any time perform smoothing directly on the decision variable. This feature suggests a heuristic application of SHAPE to a class of discrete problems. Consider a two-stage network problem where we are interested in an integer solution. We might be able to develop a piecewise linear approximation for the problem using techniques such as those described in Frantzeskakis and Powell (1990) or Powell and Cheung (1994). (Because these approximations are piecewise linear and therefore nondifferentiable, we can always satisfy the requirements of SHAPE by adding in a small nonlinear term, which is unlikely to significantly affect the final solution.) The SHAPE algorithm, then, allows us to use these approximations and produce an algorithm that still yields integer solutions that are likely to be better than the solution produced by the approximation alone.

The actual performance of SHAPE depends on its application to problems that exhibit the type of special structure that can be exploited with the initial approximation. Clearly, this question is highly problem dependent. It would be interesting to compare the empirical performance of SHAPE to the auxiliary function method of Culioli and Cohen (1990), which offers the same structural properties as SHAPE. At the same time, comparisons should be undertaken against other popular solution methods such as stochastic linearization (Gupal and Bazhenov 1972, Ermoliev 1983), stochastic decomposition (Higle and Sen 1991), and sample path optimization (Robinson 1996).

\section{ACKNOWLEDGMENTS}

This research was supported in part by grants DDM9102134 and DMI-9501446 from the National Science Foundation, and by grant AFOSR-F49620-93-1-0098 from the Air Force Office of Scientific Research, as well as the Hong Kong Research Grant Council. The authors also gratefully acknowledge the helpful comments of several anonymous referees, who helped simplify the proof of convergence as well as correct some technical issues. The authors appreciate the assistance with some technical aspects of the proof that was provided by Professor Rene Carmona in the Program for Statistics and Operations Research at Princeton University; valuable comments were also offered by Laura Wynter, Université de Versailles.

\section{REFERENCES}

Beale, E., J. Forest, C. Taylor. 1980. Multi-time period stochastic programming. In Stochastic Programming. Academic Press, New York.

Birge, J. 1985. Decomposition and partitioning techniques for multistage stochastic linear programs. Oper. Res. 33(5) 9891007.

Cheung, R., W. Powell. 1996. An algorithm for multistage dynamic networks with random arc capacities, with an application to dynamic fleet management. Oper. Res. 44(6) 951-963.

Culioli, J.-C., G. Cohen. 1990. Decomposition/coordination algorithms in stochastic optimization. SIAM J. Control and Optim. 28 1372-1403.

Doob. 1953. Stochastic Processes. John Wiley \& Sons, New York.

Ermoliev, Y. 1983. Stochastic quasigradient methods and their application to system optimization. Stochastics 9 1-36.

- 1988. Stochastic quasigradient methods. In Numerical Techniques for Stochastic Optimization. Springer-Verlag, New York.

Frantzeskakis, L., W. Powell. 1990. A successive linear approximation procedure for stochastic dynamic vehicle allocation problems. Trans. Sci. 24(1) 40-57.

Gladyshev, E. G. 1965. On stochastic approximation. Theory of Prob. and its Appl. 10 275-278.

Gupal, A.M., L.G. Bazhenov. 1972. A stochastic method of linearization. Cybernetics 482-484.

Higle, J., S. Sen. 1991. Stochastic decomposition: an algorithm for two stage linear programs with recourse. Math. Oper. Res. 16(3) 650-669.

Infanger, G. 1994. Planning under Uncertainty: Solving Largescale Stochastic Linear Programs. The Scientific Press Series, Boyd \& Fraser, New York.

Kall, P., S. Wallace. 1994. Stochastic Programming. John Wiley and Sons, New York.

Neveu, J. 1975. Discrete Parameter Martingales. North Holland, Amsterdam.

Powell, W. 1988. A comparative review of alternative algorithms for the dynamic vehicle allocation problem. In Vehicle Routing: Methods and Studies. North Holland, New York.

— R. Cheung. 1994. A network recourse decomposition method for dynamic networks with random arc capacities. Networks 24 369-384.

Robinson, S. M. 1996. Analysis of sample path optimization. Math. Oper. Res. 21(3) 1-528.

Rockafellar, T., R. Wets. 1991. Scenarios and policy aggregation in optimization under uncertainty. Math. Oper. Res. 16(1) 119-147.

Ruszczynski, A. 1980. Feasible direction methods for stochastic programming problems. Math. Programming 19 220-229.

- 1987. A linearization method for nonsmooth stochastic programming problems. Math. Oper. Res. 12(1) 32-49.

Taylor, H.M. 1990. Martingales and Random Walks. Volume 2, Elsevier Science Publishers B.V.

Van Slyke, R., R. Wets. 1969. L-shaped linear programs with applications to optimal control and stochastic programming. SIAM J. Appl. Math. 17 638-663. 